\title{
Chemical Tools for study of Phosphohistidine: Generation of Selective $\tau$-phosphohistidine and $\pi$-phosphohistidine Antibodies
}

Received 00th January 20xx, Accepted 00th January 20xx DOI: $10.1039 / x 0 x \times 00000 x$
Mehul V. Makwana, ${ }^{\text {ab }}$ Cleide dos Santos Souza, ${ }^{\mathrm{c}}$ Barry T. Pickup, ${ }^{\mathrm{a}}$ Mark J. Thompson, ${ }^{\mathrm{d}}$ Santosh K. Lomada, ${ }^{\mathrm{e}}$ Yuxi Feng, ${ }^{\mathrm{e}}$ Thomas Wieland, ${ }^{\mathrm{e}}$ Richard F. W. Jackson*a and Richmond Muimo*b

\begin{abstract}
Non-hydrolysable stable analogues of $\tau$-pHis and $\pi$-pHis have been designed using electrostatic surface potential calculations, and subsequently synthesized. The $\tau$-pHis and $\pi$-pHis analogues (phosphopyrazole 8 and pyridyl amino amide 13, respectively) were used as haptens to generate $\mathrm{pHis}$ polyclonal antibodies. Both $\tau$-pHis and $\pi$-pHis conjugates in the form of a BSA-glutaraldehyde- $\tau-\mathrm{pHis}$ and BSA-glutaraldehyde- $\pi$-pHis were synthesized and characterized by ${ }^{31} \mathrm{P}$ NMR spectroscopy. Commercially available $\tau$-pHis (SC56-2) and $\pi$-pHis (SC1-1; SC50-3) monoclonal antibodies were used to show that the BSA-G- $\tau-p H i s$ and BSA-G- $\pi$-pHis conjugates could be used to assess the selectivity of pHis antibodies in a competitive ELISA. Subsequently, the selectivity of the generated pHis antibodies generated using phosphopyrazole 8 and pyridyl amino amide 13 as haptens was assessed by competitive ELISA against His, pSer, pThr, pTyr, $\tau$-pHis and $\pi$-pHis. Antibodies generated using the phosphopyrazole $\mathbf{8}$ as a hapten were found to be selective for $\tau$-pHis, and antibodies generated using the pyridyl amino amide $\mathbf{1 3}$ were found to be selective for $\pi$-pHis. Both $\tau$ - and $\pi$-pHis antibodies were shown to be effective in immunological experiments, including ELISA, western blot, and immunofluorescence. The $\tau$-pHis antibody was also shown to be useful in the immunoprecipitation of proteins containing pHis.
\end{abstract}

methods for the detection of pHis and chemical tools which will allow

\section{Introduction}

Phosphorylation of histidine (His) residues is a post-translational modification (PTM) that occurs by formation of a phosphorus nitrogen bond (also known as a phosphoramidate bond). ${ }^{1,2}$ Unlike, the O-phosphorylated amino acid residues phosphoserine (pSer), phosphothreonine (pThr) and phosphotyrosine (pTyr), phosphohistidine (pHis) is relatively understudied which can arguably be attributed to its acid labile nature and insufficient/inadequate study tools. ${ }^{3}$ Moreover, pHis is unique, in that phosphorylation of His can occur on either one of the imidazolyl group nitrogen atoms giving rise to two isomers, namely $\tau$-pHis and $\pi$-pHis, both of which exist in nature (Fig. 1). ${ }^{2}$ There is now increasing evidence for the involvement of $\mathrm{pHis}$ in many eukaryotic cellular processes $^{3}$ and it is implicated in diseases states such as cancer. 4,5 Furthermore, there is mass spectrometry (MS) evidence that His phosphorylation is both abundant and more widely occurring than previously thought. ${ }^{6-8}$ It is therefore important to develop a range of

\footnotetext{
a. Department of Chemistry, Dainton Building, The University of Sheffield, Brook Hill, Sheffield, S3 $7 H F, U K$.

b. Department of Infection, Immunity and Cardiovascular Disease, Medical School, The University of Sheffield, Beech Hill Road, Sheffield, S10 2RX, UK.

Sheffield Institute of Translational Neuroscience, The University of Sheffield, 385a Glossop Road, Sheffield, S10 $2 \mathrm{HQ}$, UK.

d. Department of Oncology and Metabolism, Medical School, The University of Sheffield, Beech Hill Road, Sheffield, S10 2RX, UK.

e. Experimental Pharmacology, European Center of Angioscience, Medical Faculty Mannheim, Heidelberg University, 68167 Mannheim, Germany.

${ }^{*}$ Correspondence E-mail addresses: r.f.w.jackson@sheffield.ac.uk and

r.muimo@sheffield.ac.uk

†Electronic Supplementary Information (ESI) available: Experimental procedures, NMR spectra, and supplementary figures. See DOI: 10.1039/x0xx00000x ()
} for $\mathrm{pHis}$ isomer distinction, and the study of $\mathrm{pHis}$ proteins in vitro and in vivo.

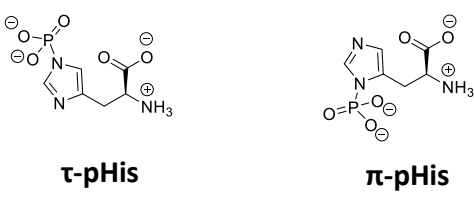

Figure 1. $\tau$-pHis and $\pi-\mathrm{pH}$ is under physiological conditions.

Antibodies have proven to be invaluable tools for studying pTyr and it is conceivable that pHis antibodies will be as effective in the study of pHis. In a 1995 review, Matthews refers to unpublished attempts to generate pHis antibodies using pHis attached to a hapten, pHis in proteins of the phosphoenol sugar transport system or phosphorylated polypHis. ${ }^{9}$ However, none of these attempts were successful in generating $\mathrm{pHis}$ antibodies; the most likely explanation was the unstable nature of pHis under physiological conditions. ${ }^{10}$ In the 1980 s a pHis cross reactive pTyr antibody was generated using azobenzylphosphonate (a proposed analogue for pTyr). ${ }^{11}$ This study was also the first to suggest that it may be possible to generate $\mathrm{pHis}$ antibodies using a non-hydrolysable pHis analogue, but a major challenge would be in designing pHis analogues that produced selective $\mathrm{pHis}$ antibodies that did not cross react with $\mathrm{pTyr} .{ }^{12}$

Several stable pHis analogues have been proposed, and some have been used in pHis antibody generation studies. ${ }^{3}$ Among these analogues, triazolyl side chains $\mathbf{3}$ and $\mathbf{6}$ have been proposed as nonhydrolysable mimics of the $\tau$-pHis $\mathbf{1}$ and $\pi$-pHis $\mathbf{5}$ side chains respectively, and have been used in the generation of $\mathrm{pH}$ is antibodies 
(Fig. 2). ${ }^{13-15}$ However, questions remain as to whether the triazolyl side chain $\mathbf{3}$ is a suitable analogue of the $\tau$-pHis side chain $\mathbf{1}$ both in antibody generation and as a chemical tool because there is experimental evidence which suggests that the triazolyl side chain $\mathbf{3}$ is a hybrid analogue of the $\tau$-pHis $\mathbf{1}$ and pTyr $\mathbf{2}$ side chains. ${ }^{3,13,14,16-18}$ In addition, it is also unclear if the triazolyl side chain $\mathbf{6}$ is an optimal analogue of the $\pi$-pHis side chain 5 because of the additional nitrogen and lone pair of electrons in the two position of the triazole ring aromatic ring.

With these considerations in mind, we proposed pyrazolyl side chain $\mathbf{4}$ as a non-hydrolysable analogue of the $\tau$-pHis side chain $1 .{ }^{19}$ Subsequently, we synthesized a pyrazolyl amino acid $\mathbf{4}$, which was used to generate pHis antibodies. ${ }^{20}$ Concurrently, in an independent report an ethylamine derivative bearing the pyrazolyl side chain 4 was also used to generate pHis antibodies. ${ }^{16}$ The pHis antibodies generated using these analogues were both found to be selective for pHis over pTyr as assessed by dot blots and sandwich ELISA. ${ }^{16,20}$

Despite the significant steps made in recent years on pHis antibody generation, the selectivity of reported $\mathrm{pHis}$ antibodies has typically been tested/determined using dot blots ${ }^{13-16,20}$ against proteins, peptides, amino acid conjugates or by sandwich ELISA ${ }^{14,16,20}$ (which is a more quantitative variant of the dot blot). The competitive ELISA, which was used to good effect to clearly show the selectivity of pTyr antibodies, ${ }^{12,21}$ is a more accurate experiment to demonstrate selectivity. The competitive ELISA used to access pTyr antibodies ${ }^{12,21}$ utilized $p$ Tyr conjugated to a carrier protein via a linker for pTyr antibody binding, in the presence of known quantities of potential competitors (e.g. phosphoamino acids ${ }^{12}$ ). Unfortunately, neither $\tau$-pHis nor $\pi$-pHis protein conjugates have hitherto been available. Furthermore, the triazolyl side chain 6 remains the only stable $\pi$-pHis analogue. ${ }^{19}$

\section{Results and discussion}

\section{Design and synthesis of $\tau$ - and $\pi$-pHis analogues}

Mapped electrostatic surface potentials (ESP) provide a visual representation of structure and charge distribution. ${ }^{22}$ Assuming molecular recognition was based on molecular shape/structure and electrostatic interaction, such representations give an insight into what the immune system is likely responding to during an immune response. Mapped ESP calculations carried out on model compounds (side chains) for $\tau$-pHis 1, pTyr 2, $\tau$-triazolyl 3, and pyrazolyl 4 side chains (Fig. 2a, middle row) confirm that the triazolyl $\mathbf{3}$ and pyrazolyl $\mathbf{4}$ side chains are structurally similar to $\tau$-pHis side chain $\mathbf{2}$ (Fig. 2a, middle row). Similarly, previously reported mapped ESP reported by Kee et al., suggested the same. ${ }^{13,16}$ However, in the mapped ESPs of

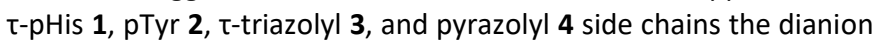
of the phosphoryl group dominates the representations and identifying subtler distinguishing features is difficult. Consequently, we considered that the electrostatic potential field could be better understood by showing more completely the variation of ESP itself (Fig. 2a, bottom row). From the ESP isocontours it was clear that for the triazolyl side chain $\mathbf{3}$, negative regions of ESP were similar to that of the pTyr side chain $\mathbf{2}$ and does not have two separate regions of negative ESP seen with the $\tau$-pHis side chain 1 . Therefore, the calculations suggested the triazolyl side chain $\mathbf{3}$ was very likely a hybrid analogue of both $\tau$-pHis and pTyr which is in line with experimental data, whereas the pyrazolyl side chain 4 has two separate regions of negative ESP separated by a $\mathrm{sp}^{2}$ hybridised carbon hydrogen bond bearing a positive ESP, analogous to the $\tau$ pHis side chain 1. It was not surprising that preserving the electronic features of a pHis analogue was paramount. For example, we previously reported that the use of a thiophene side chain (Fig. S1a) in antibody generation gave highly selective pTyr antibodies. ${ }^{12}$ Although structurally, the thiophene side chain (Fig. S1a) and the $\tau$ pHis side chain 1 appear to be similar, the ESP isocontours of the thiophene side are more closely related to the pTyr side chain $\mathbf{2}$ (Fig. S1a). Based on ESP isocontour calculations for the thiophene side chain, the comparable ESP isocontours of Schenkel's et al. proposed furan side chain analogue (Fig. S1b) of the $\tau$-pHis side chain 1 would suggest it was also a likely analogue of the pTyr side chain $\mathbf{2}$.

Comparison of model $\pi$-pHis 5 and triazolyl 6 ESP surface isocontours revealed an additional negative region in the ESP present in triazolyl side chain 6 arising from the nitrogen lone pair of electrons at the two position of the triazole aromatic ring (Fig. 2b). In the pursuit of a closer mimic of the $\pi$-pHis side $\mathbf{5}$ and inspired by the possibility of using other aromatic ring sizes (vide supra), ESP surface isocontours of a 3,4-disubstituted pyridyl side chain 7 suggested that it might be a promising analogue of $\pi$-pHis side chain 5 .

a)

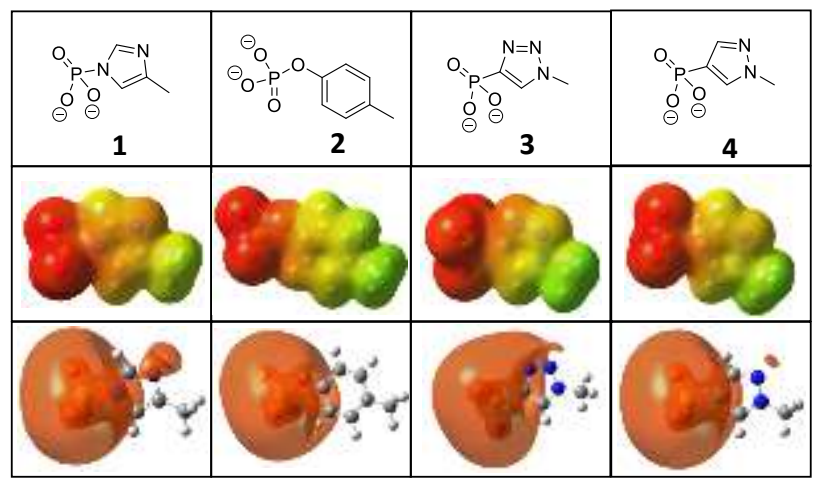

b)

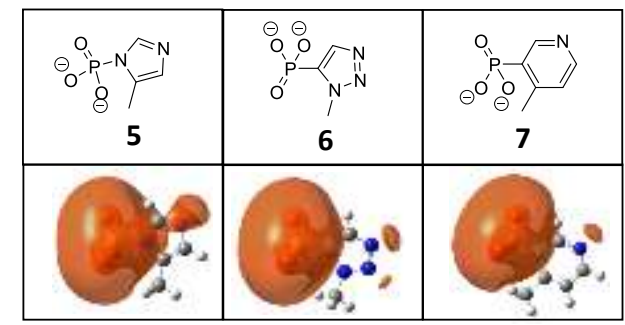

Figure 2. Density function theory calculations (DFT): a) Comparison of mapped ESP surfaces (middle row) and ESP surfaces (contours drawn at a potential of -0.285 au, bottom row) for model compounds (side chains) $\tau$-pHis 1, pTyr 2, t-triazolyl 3, and pyrazolyl 4; b) ESP surfaces (contours drawn at a potential of -0.285 au, bottom row) for model compounds $\pi$-pHis $5, \pi$-triazolyl 6, and 3,4-disubstituted pyridyl 7.

Using model side chain 7, a non-hydrolysable pyridyl amino amide $\mathbf{1 3}$ was designed as a potential stable analogue of $\pi$-pHis for use in antibody generation. The pyridyl amino amide $\mathbf{1 3}$ was specifically chosen, because historically primary amides have worked well in the generation of peptide sequence independent phosphorylated side chain binding antibodies. ${ }^{16,23,24}$ We had proposed pyrazolyl 
ethylamine $\mathbf{8}$ as a potentially useful compound for pHis antibody generation, ${ }^{19}$ and prepared it. Pyrazolyl ethylamine $\mathbf{8}$ was independently synthesised and used in pHis antibody generation. ${ }^{16}$ Despite the encouraging results of pyrazolyl ethylamine $\mathbf{8}$ in pHis polyclonal antibody generation, the selectivity of the pHis antibodies in detecting $\tau$-pHis and $\pi$-pHis isomers has not been established.

a)

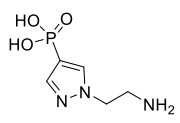

8

b)

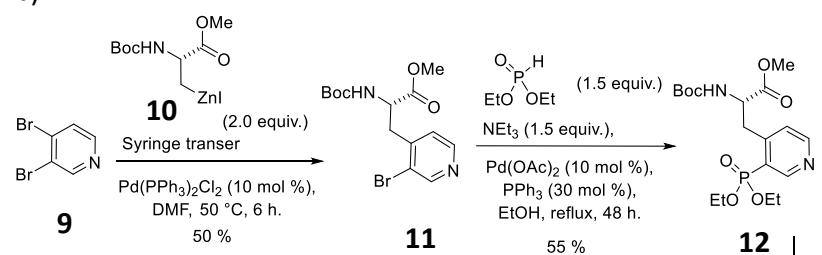

$55 \%$

12

Figure 3. Proposed non-hydrolysable $\tau$-pHis and $\pi$-pHis analogues for use in antibody generation: a) pyrazolyl ethylamine $\mathbf{8}$ analogue of $\mathrm{\tau}$-pHis (see Fig. S2 for synthesis schemes); b) synthesis scheme of non-hydrolysable pyridyl amino amide 13 analogue of $\pi$-pHis.

Pyrazolyl ethylamine $\mathbf{8}$ was synthesised following the scheme shown in Fig. S2, similar to a reported method. ${ }^{16}$ The synthesis of pyridine amino amide 13 began with selective Negishi cross coupling of zinc reagent $\mathbf{1 0}$ at the four position of 3,4-dibromopyridine 9 to give pyridine $11 .{ }^{25}$ Using the Hirao cross coupling conditions reported by Bessmertnykh et al., ${ }^{26}$ palladium catalysed cross coupling of pyridine $\mathbf{1 1}$ and diethyl phosphite gave pyridine phosphonate 12. Amidation of pyridine phosphonate 12 and subsequent treatment with $\mathrm{TMBr}$ gave the pyridine amino amide $\mathbf{1 3}$ after chromatography.

\section{Synthesis of $\tau-p H i s$ and $\pi$-pHis antibody binders; BSA-G- $\tau$-pHis and BSA-G- $\pi$-pHis conjugates}

While BSA-pTyr, BSA-pSer, and BSA-pThr conjugates are commecially available BSA-pHis conjugates are not. $\tau-\mathrm{pHis}{ }^{20} / \mathrm{pH}$ is derivatives (phosphohistamine) ${ }^{14}$ conjugated to the carrier protein BSA via a glutaraldehyde linker have previously been synthesized as $\mathrm{pHis}$ antibody binder(s), and used to assess the selectivity of pHis

antibodies by dot blots or sandwich ELISA. ${ }^{14,20}$ However, which isomeric form of pHis was conjugated to BSA or indeed whether it was a mixture of both isomers was not reported. To address this issue, samples of $\tau$-pHis and $\pi$-pHis were first synthesized by reaction of His with potassium phosphoramidate in water. ${ }^{27}$ Since $\pi$-pHis is known to undergo conversion to the more stable isomer ( $\tau$-pHis), the reaction was monitored by ${ }^{31} \mathrm{P}$ NMR spectroscopy to determine when the maximum amounts of $\tau$-pHis and $\pi$-pHis respectively, had been formed (Fig. S3 and S4). To isolate pure $\tau$-pHis and $\pi$-pHis, the crude reaction mixture was purified chromatographically ${ }^{27}$ but unlike in our previous report pHis was not precipitated. ${ }^{20}$ Instead, in order to suppress decomposition and bimolecular isomerisation of $\tau$-pHis or $\pi$-pHis it was found to be important to concentrate the combined fractions of each isomer under vacuum to a specific volume under controlled conditions ( $\left.\mathrm{pH} 10-12,25^{\circ} \mathrm{C}\right)$.

Subsequently $\tau$-pHis and $\pi$-pHis were each individually conjugated to BSA using a glutaraldehyde linker ${ }^{14,20}$ to give BSA-G- $\tau$ pHis and BSA-G- $\pi$-pHis conjugates as pHis antibody binders (Fig. 4). BSA-G- $\tau$-pHis and BSA-G- $\pi$-pHis were both stable in $0.1 \mathrm{M} \mathrm{Na}_{2} \mathrm{CO}_{3}$ $/ \mathrm{NaHCO}_{3}, \mathrm{pH} 10.8$ buffer for at least two months stored at $4{ }^{\circ} \mathrm{C}$ or $80{ }^{\circ} \mathrm{C}$ (data not included). It is worth mentioning that both in vitro and in vivo phosphorylated NME1/NME2 and PGAM were originally used to screen commercially available $\tau$-pHis and $\pi$-pHis monoclonal antibodies raised using triazolyl residues $\mathbf{3}$ and $6 .{ }^{15}$ In vitro phosphorylated NME1/NME2 and PGAM were shown to be phosphorylated on His residues by mass spectrometry analysis but the specific isomer of pHis present in NME1/NME2 and PGAM1 proteins was not directly determined but rather inferred based on reports which have shown/suggested NME1/NME2 ${ }^{28}$ to have $\pi$-pHis and PGAM1 ${ }^{29}$ to have $\tau$-pHis. ${ }^{15}$ However, some previous studies have shown that NME1 may potentially also possess $\mathrm{pSer},{ }^{30} \mathrm{pLys}$ and/or $\tau$ $\mathrm{pHis}^{31}$ and PGAM1 has $\mathrm{pTyr}^{32}$ these factors mean that potential uncertainties exist with using in vivo phosphorylated NME1 and PGAM1 as pHis standard. Hence, it was important establish that the

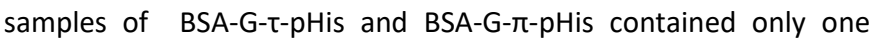
isomer, namely the isomer of pHis that had been used for their preparation (Fig. 4). While $\tau$-pHis and $\pi$-pHis are distinguishable by ${ }^{31} \mathrm{P}$ NMR spectroscopy, ${ }^{27}$ it is not always possible to distinguish reliably between $\tau$-pHis and $\pi$-pHis side chains in a protein/peptide environment using $\tau$-pHis and $\pi$-pHis ${ }^{31} \mathrm{p}$ NMR chemical shift values as references. ${ }^{33}$ On the other hand, $\tau$-pHis and $\pi$-pHis conjugated to BSA via a linker are essentially free from the influence of a protein/peptide environment. Indeed, the ${ }^{31 P}$ NMR chemical shift of

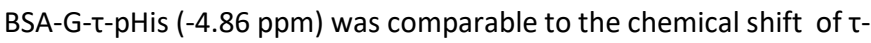
pHis (-4.78 ppm), and the chemical shift off BSA-G- $\pi-p H i s(-5.63 \mathrm{ppm})$ was comparable with the chemical shift of $\pi-p H i s$ (-5.61 ppm), (Fig. 4). Since there was no signal characteristic of $\pi-p H i s$ in BSA-G- $\tau$-pHis or $\tau-p H i s$ in BSA-G- $\pi-p H i s$ we concluded that there was no crosscontamination. To show that the BSA-G- $\tau-p H i s$ and BSA-G- $\pi-p H i{ }^{31} \mathrm{P}$ NMR signals are distinguishable, a ${ }^{31} \mathrm{P}$ NMR analysis was also carried out on a mixture of the two conjugates (Fig. 4c). The data here suggest that ${ }^{31}$ P NMR spectroscopy was particularly useful for the analyses of $\tau$-pHis and $\pi$-pHis conjugated to a protein via a linker. 


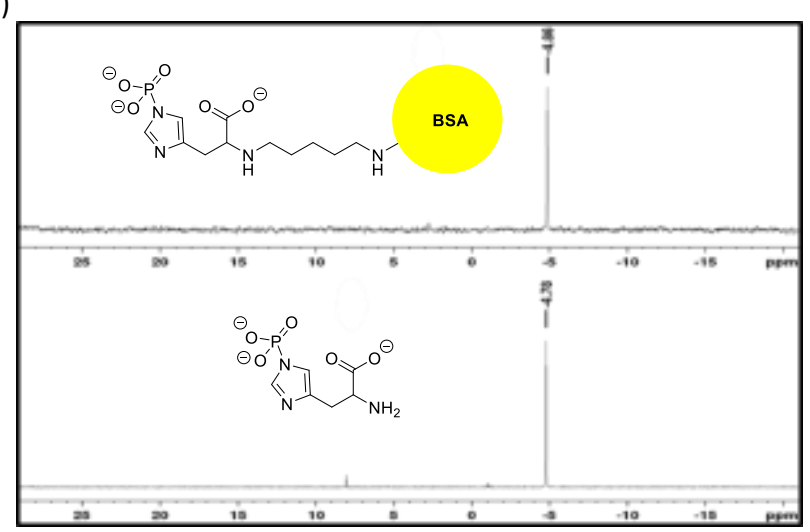

b)

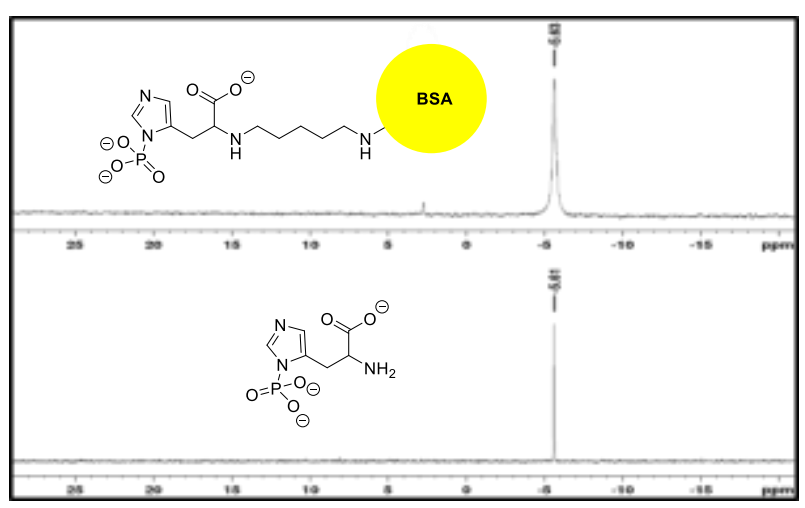

c)

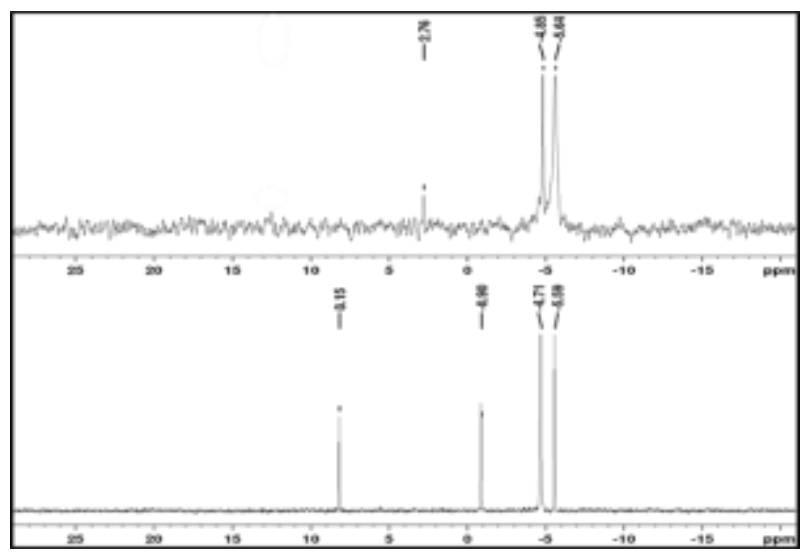

Figure 4. ${ }^{31} \mathrm{p}$ NMR spectra of $\tau-\mathrm{pH}$ is and $\pi$-pHis antibody binder conjugates: a) BSA-G- $\tau$-pHis $(35 \mathrm{mg} / \mathrm{mL})$ in $10 \%(\mathrm{v} / \mathrm{v})$ deuterium oxide, $0.1 \mathrm{M}$ $\mathrm{NaHCO}_{3} / \mathrm{Na}_{2} \mathrm{CO}_{3} \mathrm{pH} 10.8$ buffer (top spectrum) and t-pHis $(4.9 \mathrm{mg} / \mathrm{mL}$; bottom spectrum) in basic solution ( pH 12) (bottom spectrum); b) BSA-G-r$\mathrm{pHis}(40 \mathrm{mg} / \mathrm{mL})$ in $10 \%(\mathrm{v} / \mathrm{v})$ deuterium oxide, $0.1 \mathrm{M} \mathrm{NaHCO} / \mathrm{Na}_{2} \mathrm{CO}_{3} \mathrm{pH}$ 10.8 buffer (top spectrum), and $\pi$-pHis $\sim \mathrm{pH} 12(1.43 \mathrm{mg} / \mathrm{mL}$; bottom

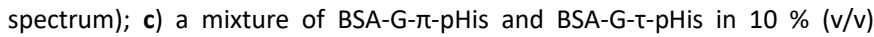
deuterium oxide, $0.1 \mathrm{M} \mathrm{NaHCO} / \mathrm{Na}_{2} \mathrm{CO}_{3} \mathrm{pH} 10.8$ buffer (top spectrum, the the minor signal at $2.75 \mathrm{ppm}$ is inorganics phosphate) and a mixture of $\pi$-pHis and $\mathrm{\tau}$-pHis (bottom spectrum) in basic solution ( pH 12). The signal $8.15 \mathrm{ppm}$ and -0.90 is an unknown impurity present in potassium phosphoramidate that was carried forward.
An external standard was used to determine the average number of pHis phosphoryl groups per BSA protein for BSA-G- $\tau-p H i s$ and BSA-G$\pi$-pHis conjugates (Table S1). An approximate molecular weight distribution analysis of BSA-G- $\tau-\mathrm{pH}$ is and BSA-G- $\pi-\mathrm{pH}$ is conjugates was determined by SDS PAGE and subsequent Coomassie staining (Fig. S5). There was a comparable distribution of monomers (BSA-GpHis), and higher molecular weight conjugates ([BSA-G-pHis $]_{n}$ ) in

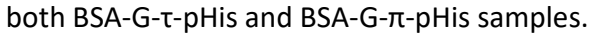

To test the applicability of BSA-G- $\tau-p H i s$ and BSA-G- $\pi-p H i s$ conjugates in a competitive ELISA, commercially available isomer specific t-pHis (SC56-2) monoclonal antibody generated using randomised combination of alanine glycine peptides containing triazolyl side chains 3, and $\pi$-pHis (SC1-1, and SC50-3) monoclonal antibodies generated using randomised combination of alanine glycine peptides containing triazolyl side chains 6 were used (Fig. 5). ${ }^{15}$ In the report which described the generation of $\tau$-pHis (SC56-2) and $\pi$-pHis (SC1-1, SC50-3) monoclonal antibodies, the selectivity of these antibodies were assessed by dot blot/western blots of peptides containing pTyr side chain $\mathbf{2}$, triazolyl side chains $\mathbf{3}$ and $\mathbf{6}$, and in vitro phosphorylated NME1/NME2 and PGAM1 but not ELISA. ${ }^{15}$ Using BSA-G- $\tau-$ pHis as the $\tau$-pHis antibody binder, the competitive ELISA profile of isomer specific $\tau$-pHis monoclonal antibody SC56-2 against amino acid competitors His, pSer, pThr, pTyr, $\tau$-pHis and $\pi$-pHis gave inconclusive results as none of the phosphoamino acids outcompeted the BSA-G- $\tau$-pHis binder (Fig 5a). In fact, the low absorption $(<0.1)$ for all amino acid's competitors would suggest the that the monoclonal antibody SC56-2 was a weak binder of the $\tau$-pHis (vide infra, compare absorbance of Fig $5 \mathrm{a}$ with $5 \mathrm{~b}$ and c; and with Fig $6 a$, and $b$ ) and thus artefactual competition was likely observed with all amino acid competitors used. Using BSA-G- $\pi$-pHis as the $\pi$-pHis antibody binder, competitive ELISA of isomer specific $\pi$-pHis monoclonal antibodies SC1-1, and SC50-3 show that both clones are selective for $\pi$-pHis (Fig. $5 b$ and c). However, at higher amino acid concentrations, competition was observed for all phosphoamino acids, in the following order: aromatic phosphoamino acids ( $\tau$-pHis and $\pi-p H i s, p T y r$ ), then hydroxy phosphoamino acids (pSer and pThr).

a)

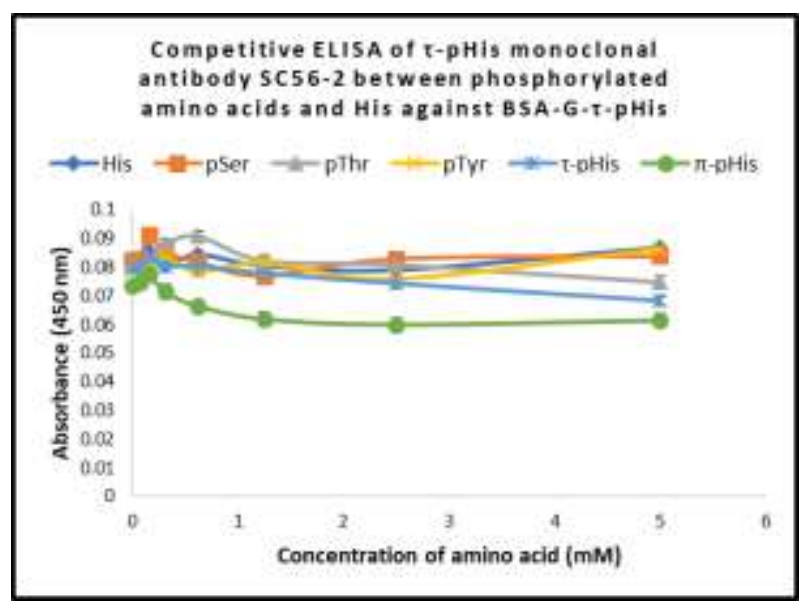


b)

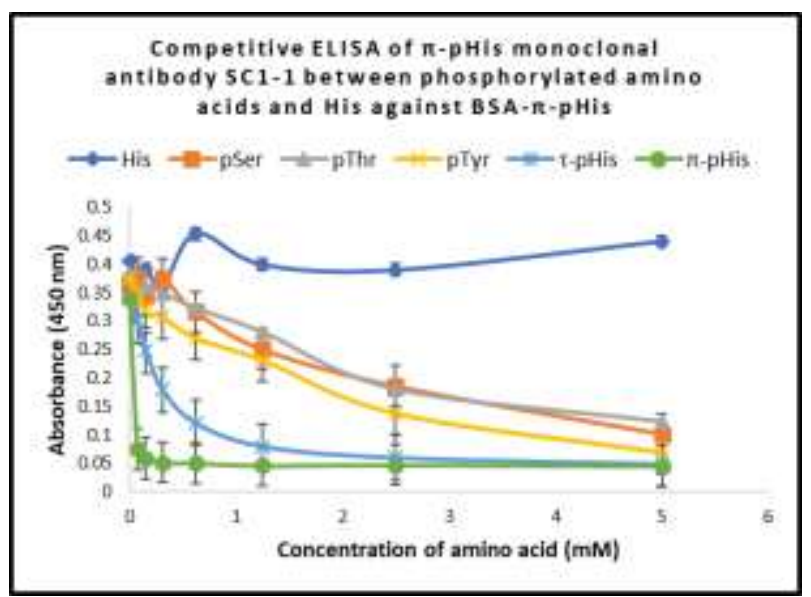

c)

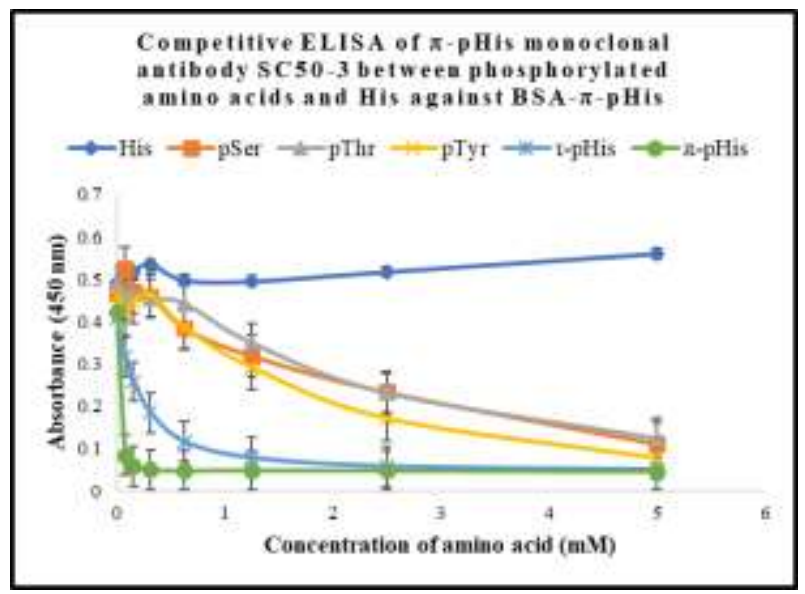

Figure 5. Competitive ELISA of commercially available pHis monoclonal antibodies with His, pSer, pThr, pTyr, $\tau-\mathrm{pH}$ is, and $\pi$-pHis against pHis antibody

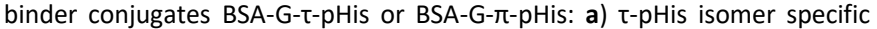
monoclonal antibody SC56-2 against BSA-G-t-pHis ; b) $\pi$-pHis isomer specific monoclonal antibody SC1-1 against BSA-G- $\pi$-pHis; c) $\pi$-pHis isomer specific monoclonal antibody SC50-3 against BSA-G- $\pi-\mathrm{pH}$ is.

\section{Generation of selective $\tau$-pHis and $\pi$-pHis polyclonal antibodies}

The combination of carrier protein $\mathrm{KLH}$, a glutaraldehyde linker and pHis analogue has previously been used in pHis antibody generation. ${ }^{16,20}$ Haptens KLH-G-8, and KLH-G-13 were prepared using standard conditions ${ }^{34}$ and administered into individual sheep. In a post immunization ${ }^{31} \mathrm{P}$ NMR spectroscopy analysis of KLH-G-13, a sharp signal at $5.02 \mathrm{ppm}$ was observed (Fig. S6). This preliminary finding suggests that in future studies, ${ }^{31}$ P NMR spectroscopy could also be used to quantify the number of phosphorylated analogues conjugated to a carrier protein, as we have shown for BSA-G- $\tau-p H i s$, and BSA-G- $\pi-p H i s$ conjugates. Sheep bleeds were collected on a monthly basis prior to boost injections, with a total of three bleeds. Analysis of the pre-immune bleed with bleeds 1-3 by sandwich ELISA indicated that both haptens had elicited an immune response with bleeds from the sheep immunized with KLH-G-8 and KLH-G-13

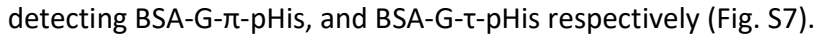

To isolate selective pHis antibodies from the antisera (bleeds 3 ) generated with KLH-G-8, and KLH-G-13, BSA-G-amino acid conjugates were used. As we have shown previously, to remove linker (glutaraldehyde) and cross-reactive antibodies, bleed 3 antisera generated with each KLH-G-8 or KLH-G-13 were each separately affinity depleted with a BSA-G-pTyr (Fig. S8) sepharose conjugate resin and the flow through was collected. ${ }^{12,20}$ To improve the selectivity of the affinity depleted antiserum generated with KLHG-8, the flow through was further affinity depleted with a BSA-G- $\pi-$ pHis sepharose conjugate resin and the second flow through was collected and labelled $\tau$-pHis-FT2. The BSA-G- $\pi-p H i s$ sepharose conjugate resin was subsequently washed and the wash was labelled $\tau-p H i s-W$. Competitive ELISA of $\tau$-pHis-FT2 and $\tau$-pHis-W showed the presence of selective $\tau$-pHis antibodies (Fig. 6a and b). Seperately, the flow through of the antiserum generated KLH-G-13 was further affinity depleted with a BSA-G- $\tau$-pHis sepharose conjugate resin and the second flow through was collected and labelled as $\pi$-pHis-FT2. The BSA-G- $\tau-p H i s$ sepharose conjugate resin was subsequently washed and the wash was labelled as $\pi$-pHis-W. Similarly, competitive ELISA of $\pi$-pHis-FT2 and $\pi-\mathrm{pHis}-\mathrm{W}$ showed the presence of selective $\pi$-pHis antibodies (Fig $6 c$ and d). Our results also demonstrate that a 6 membered phosphopyridine can be used to mimic a 5 membered aromatic ring of $\pi-p H i s$ in antibody generation. Importantly, the competitive ELISA results strongly indicate that matching electronic features (with minor compromises in structure) was likely important in designing $\mathrm{pHis}$ analogues.

On the basis of the competitive ELISA data, one could argue that the polyclonal antibodies generated using pyrazolyl ethylamine 8 and 3,4-disubstituted pyridine amino amide $\mathbf{1 3}$ are more selective and sensitive for $\tau$-pHis and $\pi$-pHis respectively, than the commercially available monoclonal antibodies which were generated using a randomised combination of alanine glycine peptides containing triazolyl side chain $\mathbf{3}$ or $\mathbf{6}$ to give $\tau$-pHis and $\pi$ pHis antibodies respectively. However, a direct comparison cannot be made between the competitive ELISA of Fig. 5 and Fig. 6 because pHis antibodies used in Fig. 5 are monoclonal and those in Fig. 6 are polyclonal. Nevertheless, the data would suggest that the pHis analogue design used in this study was effective and shows how a relatively small chemical change can have a significant effect in the generation of selective $\tau$-pHis and $\pi$-pHis polyclonal antibodies.

a)

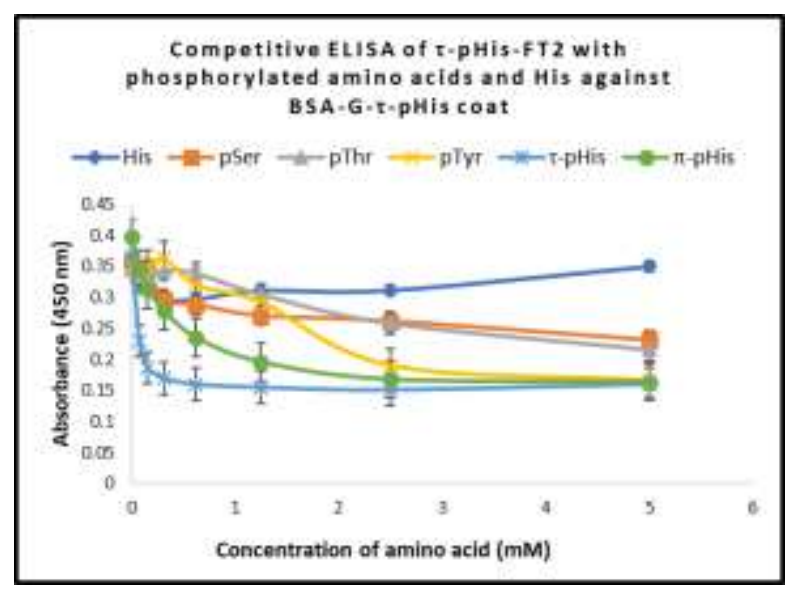


b)

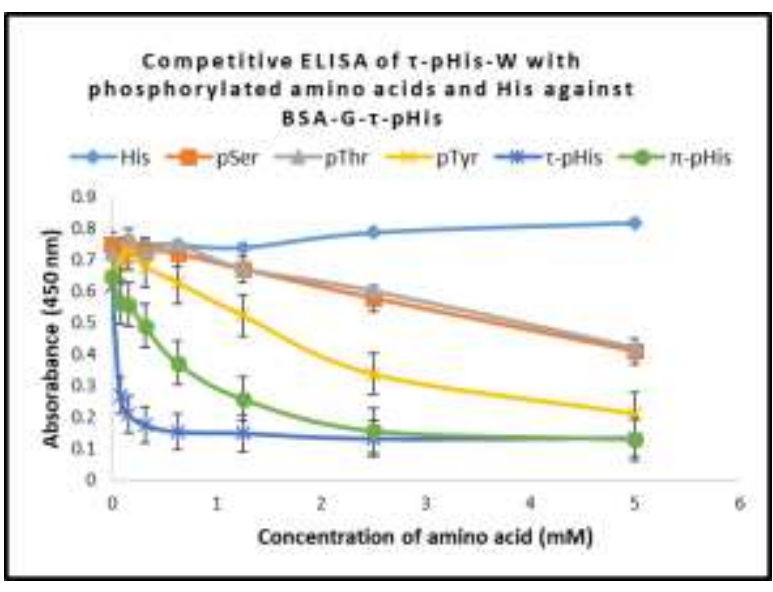

c)

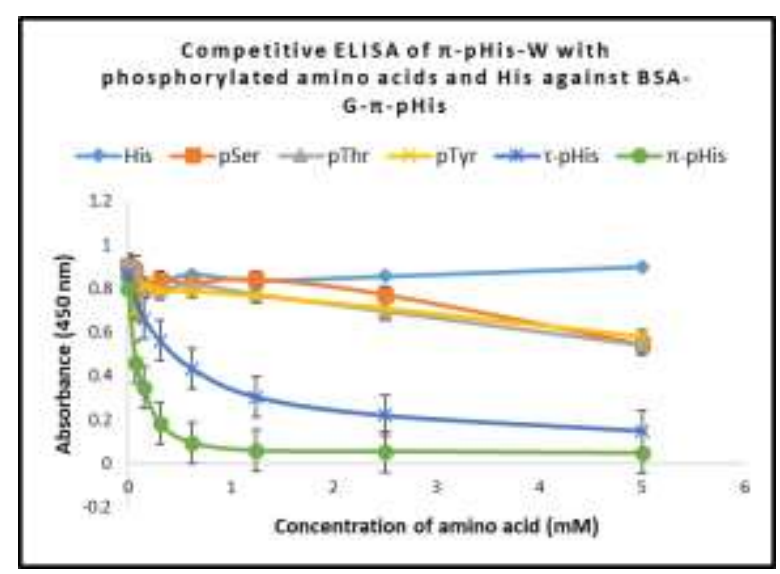

d)

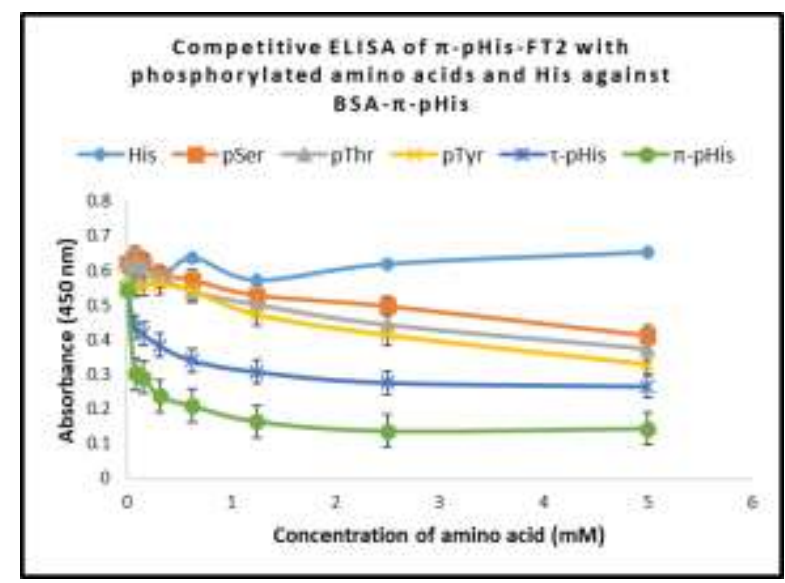

Figure 6. Competitive ELISA of polyclonal pHis antibodies with His, Ser, pThr, $\mathrm{pTyr}, \tau-\mathrm{pH}$ is and $\pi-\mathrm{pH}$ is against $\mathrm{pHis}$ antibody binder conjugates BSA-G- $\tau-\mathrm{pH}$ is

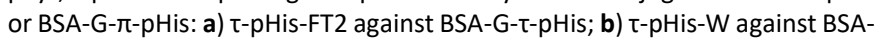

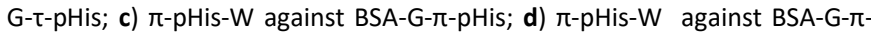
pHis.

\section{Application of $\tau$ - and $\pi$-pHis antibodies in cell biology studies}

With selective $\tau$ - and $\pi$-pHis antibodies in hand, the application of these antibodies in western blots and immunofluorescence was explored. Airway epithelia express His phosphorylated proteins and the 16HBE14o- or HBE cell line is a well-known airway epithelia cell line. ${ }^{35,36}$ Using pHis stabilizing western blot conditions (see methods) many $\mathrm{pHis}$ protein bands were detected from the 16HBE140-cell lysate using the pHis antibodies (Fig 7a-d). All pHis antibodies, $\tau$-pHisFT2, $\tau-\mathrm{pHis}-\mathrm{W}, \pi$-pHis-FT2, and $\pi$-pHis-W antibodies detected a broad molecular weight range of pHis proteins (see Fig. S9 for western blots with SC56-2, SC50-3, and SC1-1 monoclonal antibodies; only a few pHis protein bands were detected by these antibodies). Previously acidification and heating of a cell lysate prior to a western blot analysis has been used to show selective detection of pHis by pHis antibodies. ${ }^{15,20}$ Pre-acidification $(\sim \mathrm{pH} 4)$ and heating $\left(90^{\circ} \mathrm{C}, 45 \mathrm{~min}\right.$ ) of the $16 \mathrm{HBE} 14 \mathrm{o}$ - cell lysate reduced the pHis western blot signal suggesting selective detection of pHis. To further assess the generality or prevalence and potential importance of $\mathrm{pHis}$ in cellular function, the presence of $\mathrm{pHis}$ in other cell types including HUVEC and RFPEC was explored by western blots (Fig. S10 and S11). Similarly, both $\tau$-pHis-FT and $\pi$-pHis-FT antibodies detected a broad molecular weight range of pHis proteins in HUVEC and RFPEC cells. Additionally, in a qualitative analysis, immunofluorescence stained images of 16 HBE14o- cells using $\tau-p H i s-W$ and $\pi-p H i s-W$ antibodies show that pHis proteins are present throughout 16HBE14o- cells and are in higher concentrations in the nucleus than the cyctoplasm, which was more pronounced with the cells probed with the $\tau$-pHis$\mathrm{W}$ antibody (see Fig. S12 for $\tau$-pHis-W and $\pi$-pHis-W antibody dilution experiment).

Having established that $\tau-\mathrm{pHis}-\mathrm{W}$ and $\pi-\mathrm{pHis}-\mathrm{W}$ antibodies could be used in immunofluorescence imaging we also used the antibodies on other cells, namely neurons, in which pHis remains understudied. pHis has not been reported in healthy human neurons, although it has been reported in neuroblastoma cells ${ }^{37}$ and there is some indirect evidence for pHis in neurons..$^{38-40}$ Immunofluorescence stained images of human induced pluripotent stem cells (iPSCs) derived neurons probed with $\tau-\mathrm{pHis}-\mathrm{W}$ and $\pi-\mathrm{pHis}-\mathrm{W}$ antibodies shows pHis to be present throughout the cell (Fig. 8b). However, in contrast to the 16HBE14o- cells, only a weak pHis immunofluorescence staining was observed in the neuron nucleus. Due to paucity of material, it was not possible to analyse the pHis proteins from the neurons by western blots. The presence of $\mathrm{pHis}$ proteins in human iPSCs derived neurons is especially interesting because protein phosphorylation (O-linked) is implicated/involved in many neurological disease states. ${ }^{41}$ This therefore raises some important questions: what is the biological function of $\mathrm{pHis}$ in neurons; is pHis involved in neurological diseases; what is the biological relevance of little/no $\mathrm{pHis}$ in the neuron nucleus compared to 16HBE14o- and Muller cells (vide infra)?

Furthermore, Muller wild type (WT) and Muller nucleoside diphosphate kinase B (NDPK-B) knockout (KO) cells ${ }^{42}$ were analysed by immunofluorescence staining for $\mathrm{pHis}$ using $\tau-\mathrm{pHis}-\mathrm{W}$ and $\pi-\mathrm{pHis}-$ $W$ antibodies (red). Interestingly, though NDPK-B is a known mammalian $\mathrm{His}$ kinase ${ }^{43}$ which is involved in many cellular functions ${ }^{44}$ there was no manifestly clear difference between the immunofluorescence intensities of Muller WT and NDPK-B KO cells probed with the $\tau$-pHis-W antibody (Fig. S13a). 
a)

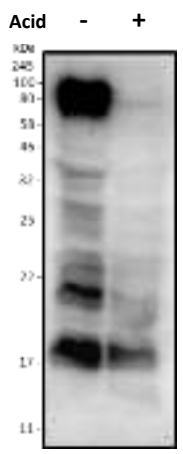

t-pHis-FT2

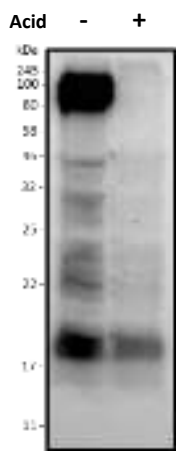

t-pHis-W

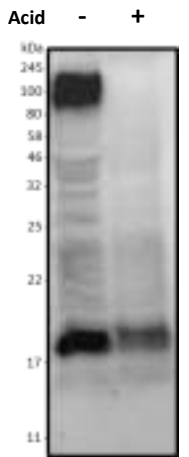

$\pi-$ pHis-FT2

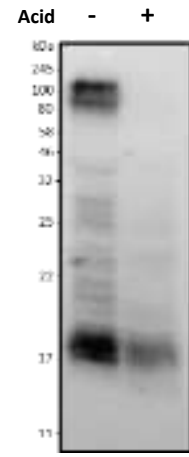

$\pi-p H i s-W$
Figure 7. Western blot of $16 \mathrm{HBE} 14 \mathrm{o}$-cell lysate probed with pHis polyclona antibodies untreated (left lane), and after treatment with acetic acid to $\sim \mathrm{pH}$

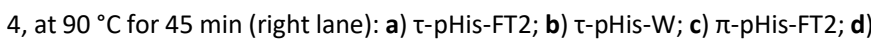
$\pi-\mathrm{pHis}-\mathrm{W}$.

However, there was a noticeable increase in immunofluorescence intensity of Muller NDPK-B KO cells compared to Muller WT cells probed with the $\pi$-pHis-W antibody (Fig S13b). These observations were also reflected in western blots of the Muller WT vs Muller NDPK $B$ KO cells (Fig. S14). The $\pi-p H i s-W$ antibody can detect in vitro phosphorylated NDPK-A, B, and C (Fig. S15), ${ }^{45}$ and thus one might have expected a weaker $\pi$-pHis immunofluorescence signal from the NDPK-B KO Muller cells compared to the WT because NDPK-B is thought to be phosphorylated on a His residue to give $\pi$-pHis. ${ }^{3} \mathrm{~A}$ plausible explanation could be the upregulation of some $\pi$-pHis proteins to compensate for the absence of NDPK-B.

a)

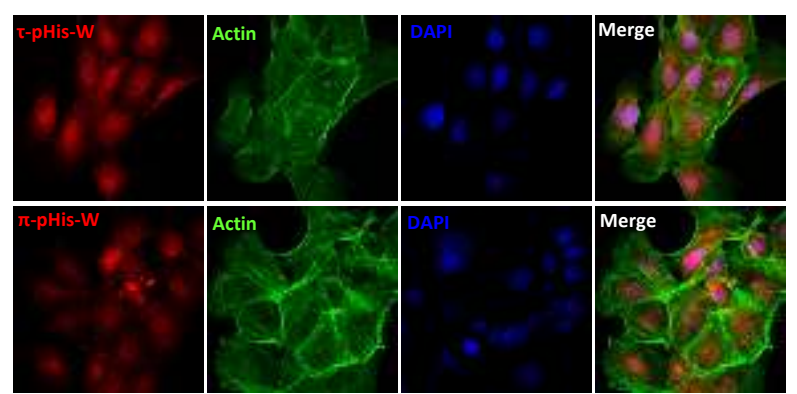

b)

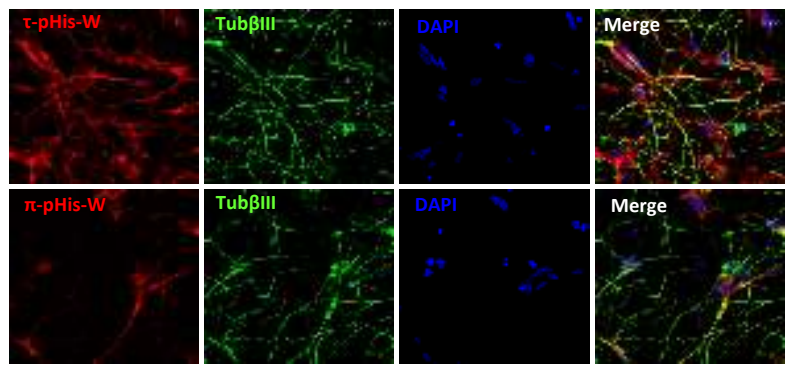

c)

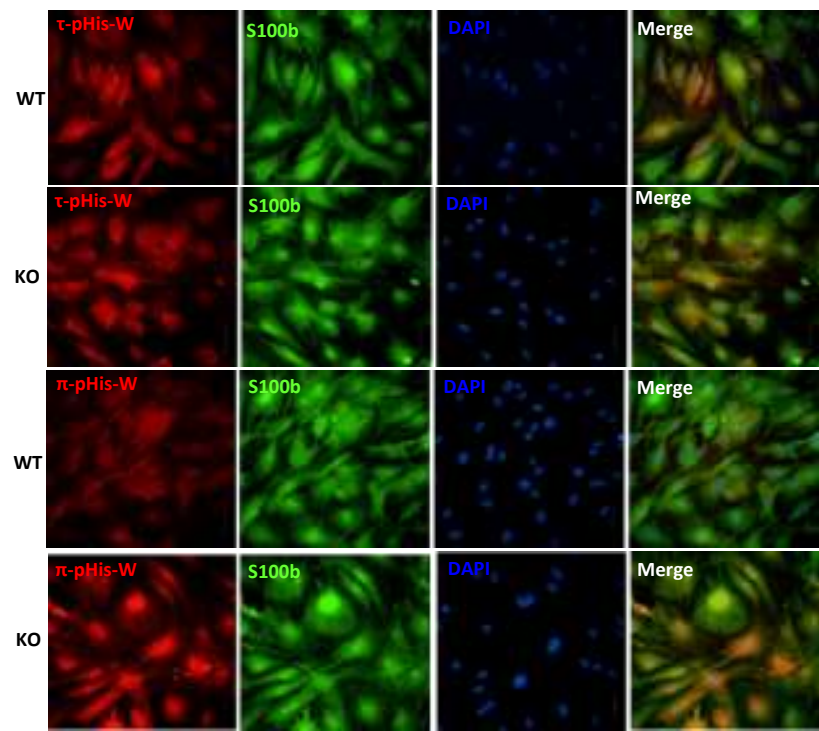

Figure 8. Immunofluorescence images using $\tau-p H i s-W$ and $\pi-p H i s-W$ antibodies of: a) $16 \mathrm{HBE} 140$ - cells stained using $\tau$-pHis-W and $\pi-\mathrm{pHis}-\mathrm{W}$ antibodies (red, Alexa Fluor ${ }^{\mathrm{TM}} 594$ secondary antibody), Alexa Fluor ${ }^{\mathrm{TM}} 488$ Phalloidin (Green) that stain actin filaments was used as a marker that define the boundary of cells and DAPI (blue) for nuclear staining.; b) Human induced pluripotent stem cells (iPSCs) derived neurons stained wit: - - citis-W and $\pi$ pHis-W antibody (red, Alexa Fluor ${ }^{\mathrm{TM}} 594$ secondary antibody), TubßIII (green, Alexa Fluor ${ }^{\mathrm{TM}} 488$ secondary antibody) a classical neuronal marker and DAPI (blue); c) Muller wild type (WT) and Muller NDPK-B knockout (KO) cells stained with $\tau$-pHis-W and $\pi-p H i s-W$ antibody (red, Alexa Fluor ${ }^{\mathrm{TM}} 594$ secondary antibody), S100b (green, Alexa Fluor ${ }^{\mathrm{TM}} 488$ secondary antibody) a glial cell marker, and DAPI (blue).

To demonstrate that the $\mathrm{pHis}$ antibodies could potentially be used as a pHis protein enrichment tool, the $\tau$-pHis-FT2 antibody was used in immunoprecipitation experiments (the $\tau$-pHis-FT2 antibody was chosen as it was available in the largest quantities). The $\tau$-pHis-FT2 antibody immobilized on protein $\mathrm{G}$ sepharose was used to pull down proteins from the 16HBE140- cell lysate. After the washes, bound proteins were competitively eluted using a synthesized phosphopyrazolyl butane $\mathbf{1 4}$ (see Fig. S16 for synthesis scheme). $\tau$ $\mathrm{pHis}$ or $\pi$-pHis were considered not suitable for use as competitors due to their tendency to transfer the phosphoryl group to other nucleophilic residues which could give rise to false positives. Neither could pyrazolyl ethylamine 8 be used because primary amines are known to dephosphorylate pHis derivatives. ${ }^{46}$ The eluted fractions were examined by colloidal gold protein stain and western blot using the $\tau$-pHis-FT2 antibody (Fig. S17). The competitively extracted fractions gave reduced colloidal gold staining and pHis western blot signal when the proteins from the 16HBE14o- cell lysate was treated with acid prior to immunoprecipitation. The data here would suggest pHis proteins were indeed being immunoprecipitated by the $\tau$-pHisFT2.

\section{Conclusions}

This study demonstrates the importance of a chemical level approach in the rational design of $\tau-p H i s$ and $\pi$-pHis analogues and their application in $\tau$-pHis and $\pi$-pHis antibody generation. 
Application of chemical design guided by ESP isocontour calculations, provided pyrazolyl ethylamine $\mathbf{8}$ and pyridyl amino amide $\mathbf{1 3}$ which were used as haptens to generate selective $\tau$-pHis and $\pi$-pHis polyclonal antibodies respectively. The application of the $\tau-\mathrm{pHis}$ and $\pi$-pHis polyclonal antibodies in ELISA, western blot, immunofluorescence experiments and immunoprecipitation demonstrated their broad applicability in common biological experiments. The results of these preliminary biological experiments suggested that $\mathrm{pHis}$ is a significant and widespread PTM in mammalians cells and requires further investigation. Consequently, the $\mathrm{pH}$ is analogues developed in this study including the BSA-G- $\tau$ $\mathrm{pHis}$ and BSA-G- $\pi-\mathrm{pH}$ is conjugates will be very useful in monoclonal antibody generation and selection and other cell biological studies. Other pHis analogues, and $\mathrm{pHis}$ analogues which can be incorporated into peptides as well as the application of pyrazolyl ethylamine $\mathbf{8}$ and pyridyl amino amide $\mathbf{1 3}$ in the generation of $\tau$-pHis and $\pi$-pHis monoclonal antibodies is currently under way.

\section{Conflicts of interest}

Two of the authors are named on relevant patents (WO/2015/033120; EU - 20160207948 and USA - US 10,053,476).

\section{Acknowledgements}

We would like to thank Patrik Niesobski for preliminary synthetic work; Bezaleel Mambwe for technical assistance; Sandra van Meurs and Mike P. Williamson for ${ }^{31} \mathrm{P}$ NMR spectroscopy assistance; Anupriya Chatterjee for providing HUVEC cells, and EPSRC for support (DTA studentship to M.V.M.).

\section{Notes and references}

1 P. V. Attwood; M. J. Piggott; X. L. Zu and P. G. Besant, Amino Acids, 2007, 32, 145.

2 S. Klumpp and J. Krieglstein, Eur. J. Biochem., 2002, 269, 1067.

3 M. V. Makwana; R. Muimo and R. F. W. Jackson, Lab. Invest., 2017, 98, 291.

4 S. K. Hindupur; M. Colombi; S. R. Fuhs; M. S. Matter; Y. Guri; K. Adam; M. Cornu; S. Piscuoglio; C. K. Y. Ng; C. Betz; D. Liko; L. Quagliata; S. Moes; P. Jenoe; L. M. Terracciano; M. H. Heim; T. Hunter and M. N. Hall, Nature, 2018, 555, 678.

5 I. Khan and P. S. Steeg, Oncotarget, 2018, 9, 10185.

6 G. Hardman; S. Perkins; P. J. Brownridge; C. J. Clarke; D. P. Byrne; A. E. Campbell; A. Kalyuzhnyy; A. Myall; P. A. Eyers; A. R. Jones and C. E. Eyers, EMBO J., 2019, 38, e100847.

7 Y. Gao; H. Lee; O. K. Kwon; Z. Cheng; M. Tan; K.-T. Kim and S. Lee, Proteomics, 2019, 19, 1800471.

8 C. M. Potel; M.-H. Lin; A. J. R. Heck and S. Lemeer, Nat. Methods, 2018, 15, 187.

9 H. R. Matthews, Pharmacol. Ther., 1995, 67, 323.

10 D. E. Hultquist, Biochim. Biophys. Acta, 1968, 153, 329.

11 A. R. Frackelton; A. H. Ross and H. N. Eisen, Mol. Cell. Biol., 1983, 3, 1343 .

12 M. Lilley; B. Mambwe; R. F. W. Jackson and R. Muimo, Chem. Commun., 2014, 50, 9343.

13 J.-M. Kee; B. Villani; L. R. Carpenter and T. W. Muir, J. Am. Chem. Soc., 2010, 132, 14327.
14 J. M. Kee; R. C. Oslund; D. H. Perlman and T. W. Muir, Nat. Chem. Biol., 2013, 9, 416

15 S. R. Fuhs; J. Meisenhelder; A. Aslanian; L. Ma; A. Zagorska; M. Stankova; A. Binnie; F. Al-Obeidi; J. Mauger; G. Lemke; J. R. Yates, 3rd and T. Hunter, Cell, 2015, 162, 198.

16 J.-M. Kee; R. C. Oslund; A. D. Couvillon and T. W. Muir, Org. Lett., 2015, 17, 187.

17 L. Senderowicz; J. X. Wang; L. Y. Wang; S. Yoshizawa; W. M. Kavanaugh and C. W. Turck, Biochemistry, 1997, 36, 10538.

18 T. E. McAllister; K. A. Horner and M. E. Webb, ChemBioChem, 2014, 15, 1088.

19 R. Jackson; R. Muimo and M. Lilley WO 2015/033120 Al, 2015.

20 M. Lilley; B. Mambwe; M. J. Thompson; R. F. W. Jackson and R. Muimo, Chem. Commun., 2015, 51, 7305.

21 J. R. Glenney; L. Zokas and M. P. Kamps, J. Immunol. Methods, 1988, 109, 277.

22 G. Naray-Szabo and G. G. Ferenczy, Chem. Rev., 1995, 95, 829.

23 A. R. Frackelton; M. Posner; B. Kannan and F. Mermelstein, Methods Enzymol., 1991, 201, 79.

24 H. Ouyang; C. Fu; S. Fu; Z. Ji; Y. Sun; P. Deng and Y. Zhao, Org. Biomol. Chem., 2016, 14, 1925.

25 S. Tabanella; I. Valancogne and R. F. W. Jackson, Org. Biomol. Chem., 2003, 1, 4254.

26 A. Bessmertnykh; C. M. Douaihy; S. Muniappan and R. Guilard, Synthesis-Stuttgart, 2008, 1575.

27 P. G. Besant; L. Byrne; G. Thomas and P. V. Attwood, Anal. Biochem., 1998, 258, 372.

28 S. Morera; I. Lascu; C. Dumas; G. Lebras; P. Briozzo; M. Veron and J. Janin, Biochemistry, 1994, 33, 459.

29 M. G. Vander Heiden; J. W. Locasale; K. D. Swanson; H. Sharfi; G. J. Heffron; D. Amador-Noguez; H. R. Christofk; G. Wagner; J. D. Rabinowitz; J. M. Asara and L. C. Cantley, Science, 2010, 329, 1492.

30 N. J. Macdonald; A. Delarosa; M. A. Benedict; J. M. P. Freije; H. Krutsch and P. S. Steeg, J. Biol. Chem., 1993, 268, 25780.

31 O. Walinder, J. Biol. Chem., 1968, 243, 3947.

32 T. Hitosugi; L. Zhou; J. Fan; S. Elf; L. Zhang; J. Xie; Y. Wang; T.-L. Gu; M. Alečković; G. LeRoy; Y. Kang; H.-B. Kang; J.-H. Seo; C. Shan; P. Jin; W. Gong; S. Lonial; M. L. Arellano; H. J. Khoury; G. Z. Chen; D. M. Shin; F. R. Khuri; T. J. Boggon; S. Kang; C. He and J. Chen, Nat. Commun., 2013, 4, 1790.

33 A. Lecroisey; I. Lascu; A. Bominaar; M. Veron and M. Delepierre, Biochemistry, 1995, 34, 12445.

34 G. T. Hermanson Bioconjugate Techniques, 2nd Edition; Elsevier London, 2008.

35 R. Muimo; S. J. Banner; L. J. Marshall and A. Mehta, Am. J. Respir. Cell Mol. Biol., 1998, 18, 270.

36 R. Muimo; Z. Hornickova; C. E. Riemen; V. Gerke; H. Matthews and A. Mehta, J. Biol. Chem., 2000, 275, 36632.

37 K. Adam; J. Lesperance; T. Hunter and P. E. Zage, Int. J. Mol. Sci., 2020, 21.

38 S. Klumpp; J. Hermesmeier; D. Selke; R. Baumeister; R. Kellner and J. Krieglstein, J. Cerebr. Blood F. Met., 2002, 22, 1420.

39 C.-W. Chen; H.-L. Wang; C.-W. Huang; C.-Y. Huang; W. K. Lim; I.C. Tu; A. Koorapati; S.-T. Hsieh; H.-W. Kan; S.-R. Tzeng; J.-C. Liao; W. M. Chong; I. Naroditzky; D. Kidron; A. Eran; Y. Nijim; E. Sela; H. B. Feldman; L. Kalfon; H. Raveh-Barak; T. C. Falik-Zaccai; O. Elpeleg; H. Mandel and Z.-F. Chang, Proc. Natl. Acad. Sci., 2019, 116, 566.

40 M. Carotenuto; E. Pedone; D. Diana; P. de Antonellis; S. Džeroski; N. Marino; L. Navas; V. Di Dato; M. N. Scoppettuolo; F. Cimmino; S. Correale; L. Pirone; S. M. Monti; E. Bruder; B. Zenko; I. Slavkov; F. Pastorino; M. Ponzoni; J. H. Schulte; A. Schramm; A. Eggert; F. 
Westermann; G. Arrigoni; B. Accordi; G. Basso; M. Saviano; R. Fattorusso and M. Zollo, Sci. Rep., 2013, 3, 1351.

41 S. Tenreiro; K. Eckermann and T. F. Outeiro, Front. Mol. Neurosci., 2014, 7, 42.

42 L. Di; S. Srivastava; O. Zhdanova; Y. Sun; Z. Li and E. Y. Skolnik, J. Biol. Chem., 2010, 285, 38765.

43 F. Cuello; R. A. Schulze; F. Heemeyer; H. E. Meyer; S. Lutz; K. H. Jakobs; F. Niroomand and T. Wieland, J. Biol. Chem., 2003, 278, 7220. 44 P. V. Attwood and R. Muimo, Lab. Invest., 2018, 98, 283.

45 F. Buljubasic; I. El-Battrawy; H. Lan; S. K. Lomada; A. Chatterjee; Z. Zhao; X. Li; R. Zhong; Q. Xu; M. Huang; Z. Liao; S. Lang; L. Cyganek; X. Zhou; T. Wieland; M. Borggrefe and I. Akin, J. Clin. Med., 2020, 9, 486.

46 W. P. Jencks and M. Gilchrist, J. Am. Chem. Soc., 1965, 87, 3199. 